\title{
EFFICACY OF SILODOSIN VS TAMSULOSIN IN PATIENTS WITH BENIGN PROSTATIC HYPERPLASIA: A DOUBLE-BLIND RANDOMIZED CLINICAL TRIAL
}

\section{S. T. CAHYO ARIWICAKSONO1* ${ }^{*}$, ROBERTUS B. PRASETYO ${ }^{2}$, NINDRA PRASADJA, NUGROHO B. UTOMO}

1Department of Urology, Faculty of Medicine Universitas Indonesia, 2Urology Division, Surgery Department, Indonesian Army Hospital RSPAD Gatot Soebroto

Email: cahyoariwicaksono@gmail.com

Received: 12 Dec 2018, Revised and Accepted: 10 Mar 2019

\section{ABSTRACT}

Objective: Benign prostatic hyperplasia (BPH) is a nonmalignant enlargement of the prostate associated with aging. BPH can cause lower urinary tract syndrome (LUTS). Medical therapy for patients with moderate and severe LUTS symptoms comprises $\alpha-1$ adrenergic receptor antagonists. This study aimed to determine whether there are differences in the international prostate symptom score (IPSS) and maximal flow rate (Qmax) of patients with BPH receiving either silodosin or tamsulosin over $12 \mathrm{w}$.

Methods: This study was a double-blind randomized clinical trial. Subjects were 50 men aged $\geq 50$ y diagnosed with BPH with an IPSS $\geq 8$ at the Gatot Soebroto Indonesian Army Hospital. The participants received either silodosin or tamsulosin. Their IPSS and Qmax were assessed at the initial assessment and after 4,8 , and $12 \mathrm{w}$ of treatment.

Results: The initial median IPSS was 15 in the tamsulosin group and 17 in the silodosin group $(P=0.808)$. After $12 \mathrm{w}$ of therapy, the median IPSS decreased to 9 in the tamsulosin group and 10 in the silodosin group $(P=0.186)$. The initial median $Q_{\max }$ was $10.1 \mathrm{ml} / \mathrm{s}$ in the tamsulosin group and $10.9 \mathrm{ml} / \mathrm{s}$ in the silodosin group $(P=0.290)$. After $12 \mathrm{w}$ of therapy, the median $\mathrm{Q}_{\max }$ increased to $12.1 \mathrm{ml} / \mathrm{s}$ in the tamsulosin group and $11.9 \mathrm{ml} / \mathrm{s}$ in the silodosin group $(P=0.969)$. Although the differences between groups were not significant, the initial and 12-week IPSS and $Q_{\text {max }}$ values differed significantly within each group.

Conclusion: There were no significant between-group differences in the IPSS or $\mathrm{Q}_{\max }$ after $12 \mathrm{w}$ of therapy. However, both silodosin and tamsulosin produced significant differences between initial and 12-week assessments of IPSS and $Q_{\max }$.

Keywords: Benign prostate hyperplasia, Alpha-blocker, IPSS, Maximal flow rate

(C) 2019 The Authors. Published by Innovare Academic Sciences Pvt Ltd. This is an open access article under the CC BY license (http://creativecommons. org/licenses/by/4. 0/) DOI: http://dx.doi.org/10.22159/ijap.2019.v11s6.33558

\section{INTRODUCTION}

Benign prostatic hyperplasia $(\mathrm{BPH})$ prevalence in men increases with age. At 50 y old, $50 \%-75 \%$ of men will suffer from BPH. This proportion increases to $80 \%$ in men aged 70 y or more, and to $88 \%$ $90 \%$ in men aged 81 y and over [1,2]. The prevalence of BPH in Indonesia has not been reported. The prevalence in Cipto Mangunkusumo General Hospital from 1994 to 2013 was 3804 patients with a mean age of $66.61 \mathrm{y}$ [1].

Patients with BPH have symptoms of lower urinary tract syndrome (LUTS). LUTS symptoms are divided into obstructive, irritative, and postvoiding symptoms. Obstructive symptoms include a weak urine stream, interrupted voiding, straining to urinate, and retention of urine; irritative symptoms include polyuria, dysuria, urgency, and nocturia; and the postvoiding symptom is dribbling urine [1-3]. Platz reported in 2012 that the prevalence of moderate and severe LUTS was 41 and 19 cases per 1000 patients/year, respectively [4].

The international prostate symptom score (IPSS) is recommended as an ideal instrument for screening the severity of LUTS, the therapeutic response, and improvement in symptoms. Patients with moderate and severe LUTS (IPSS 8-35) require additional evaluations including a urodynamic test, uroflowmetry, and evaluation of postvoiding urinary retention [5].

Medical therapy with an $\alpha-1$ adrenergic receptor antagonist ( $\alpha 1-\mathrm{AR})$ is usually given to patients with moderate and severe LUTS $[1,3,6-$ 8]. These drugs inhibit smooth muscle contraction in the prostate, and thus decrease the resistance of the bladder neck and improve urethral tone. The first generation of $\alpha 1$-ARs used to treat patients with BPH were nonselective. Subsequently, selective $\alpha 1$-ARs including tamsulosin and naftopidil were developed.

Recently, a more selective $\alpha 1-\mathrm{AR}$, silodosin, was developed in Japan. Silodosin was confirmed to be 38 times more selective toward $\alpha 1 \mathrm{~A}$ adrenergic receptors than $\alpha 1 B$ adrenergic receptors $[9,10]$. A phase
III clinical trial found that silodosin improved the IPSS within the first $2 \mathrm{w}$ of usage [7], and another trial confirmed that the efficacy of 8 $\mathrm{mg} /$ day silodosin was comparable to that of $0.2 \mathrm{mg} /$ day tamsulosin [10]. Few trials have compared silodosin with tamsulosin in clinical practice [9]. The present study aimed to compare the efficacy of 8 $\mathrm{mg}$ /day silodosin with that of $0.4 \mathrm{mg}$ /day tamsulosin in patients with $\mathrm{BPH}$ with moderate-severe LUTS symptoms in a double blind randomized clinical trial. Because clinical use of silodosin was only recently initiated in Indonesia, this study was considered as a pioneer study of the efficacy of silodosin in BPH.

\section{MATERIALS AND METHODS}

This was a double-blind clinical trial that was conducted in the Urology Clinic, Gatot Subroto Central Army Hospital. Data were gathered from February 2017 to July 2017. The Faculty of Medicine, Universitas Indonesia Ethics Committee approved the study protocol (ethics clearance number 382/UN2. F1/ETIK/2017). Consecutive sampling was used in this study with a total sample size of 50 subjects who were divided into two groups. The inclusion criteria were men aged $\geq 50 \mathrm{y}$ who were diagnosed with LUTS related to BPH, had an IPSS $\geq 8$, a $\mathrm{Q}_{\max }>4$ and $<15 \mathrm{ml} / \mathrm{s}$, and who had agreed to complete the 12-week study. Patients with prostate cancer or who refused silodosin or tamsulosin were excluded from the study. To ensure blinding, a third party was assigned to allocate the treatment arm using similar sealed envelopes. Therefore, neither the investigators nor the patients knew which drug was given to the patients.

Patients that fulfilled the inclusion criteria and had not been excluded from the Urology Clinic in Gatot Subroto Central Army Hospital were the ones who underwent a one-week washout from their previous therapy. When the LUTS symptoms appeared after one week, IPSS and uroflowmetry assessments were performed, after which patients were allocated to silodosin $8 \mathrm{mg} /$ day or tamsulosin $0.4 \mathrm{mg}$ /day using the double-blind procedure. Assessment of IPSS and $\mathrm{Q}_{\max }$ were performed after 4,8 , and $12 \mathrm{w}$ of treatment. 
The variables collected in this study were identity, age, IPSS, and uroflowmetry results. The data gathered are presented descriptively and analytically. Descriptive analysis was used for the subjects' demographic characteristics including age, IPSS score, and uroflowmetry. The data are presented as mean \pm standard deviation (SD) or as median (min-max) if the data were not normally distributed.

We also compared the efficacy of silodosin and tamsulosin. Comparative analysis of IPSS and Qmax within each drug-treatment group were performed during the initial assessment, and after 4, 8, and $12 \mathrm{w}$ of treatment. A repeated-measures analysis of variance with Bonferroni post hoc correction was employed if the data were normally distributed or could be $\log 10$ transformed. If the data were not normally distributed, Friedman's test and the Wilcoxon post hoc test were employed.

Analyses comparing the IPSS and $Q_{\max }$ for silodosin and tamsulosin were performed at the initial assessment and after 4,8 , and $12 \mathrm{w}$ of treatment. A generalized linear model was employed if the data were normally distributed or the data were $\log _{10}$ transformed. If the data were not normally distributed, a repeated-measures MannWhitney test was used.
Differences were deemed to be significant if $P<0.05$. The software employed in this study was the Statistical Package for the Social Sciences (SPSS) ver. 20 for Windows by IBM (address: New York, USA). Ethical issues that could arise in this study included patient confidentiality and drug-related adverse events. Patient medical records were considered confidential and the access to the medical records was limited to the investigators. At the beginning of the study, the subjects were informed about the potential adverse events that could occur, including ejaculatory disorders (retrograde ejaculation), increased risk of upper respiratory tract infection, and thirst. All subjects were also advised to mention any adverse events at every visit $(4,8$, and $12 \mathrm{w})$ so that they could be recorded.

\section{RESULTS}

Fifty subjects participated in this clinical trial. Subjects were divided into tamsulosin and silodosin groups. The median age of subjects in the tamsulosin group was 61.8 (53-73) years and that of the silodosin group was 61.7 (53-72) years. The demographic characteristics of the subjects are listed in table 1.

Table 1: Demographic and clinical characteristics of subjects

\begin{tabular}{lllll}
\hline & N & Tamsulosin median (min-max) & N & Silodosin median (min-max) \\
\hline Age & 25 & $61(53-73)$ & 25 & $63(53-72)$ \\
Initial IPSS & 25 & $15(9-29)$ & 25 & $17(8-26)$ \\
Initial Q $\max$ & 25 & $10.1(4.1-13.4)$ & 25 & $10.9(1.9-19.8)$ \\
\hline
\end{tabular}

IPSS = international prostate symptom score., In the tamsulosin group, there was a significant reduction in IPSS $(P=<0.001)$ and a significant increase in $\mathrm{Q}_{\max }(P=<0.001)$ between the initial and 12-week assessment (table 2).

Table 2: Effect of tamsulosin on IPSS and uroflowmetry

\begin{tabular}{|c|c|c|c|c|}
\hline & & $\mathbf{N}$ & Median (min-max) & $\boldsymbol{P}$ \\
\hline \multirow[t]{4}{*}{ IPSS } & Initial & 25 & $15(9-29)$ & \multirow[t]{4}{*}{$<0.001$} \\
\hline & $4 \mathrm{w}$ & 25 & $11(8-22)$ & \\
\hline & $8 w$ & 25 & $9(7-17)$ & \\
\hline & $12 \mathrm{w}$ & 25 & $9(7-15)$ & \\
\hline Initial vs $12 \mathrm{w}$ IPSS & & & & $<0.001$ \\
\hline$Q_{\max }$ & Initial & 25 & $10.1(4.1-13.4)$ & $<0.001$ \\
\hline$(\mathrm{ml} / \mathrm{s})$ & $4 \mathrm{w}$ & 25 & $11.5(5.7-14.5)$ & \\
\hline & $8 w$ & 25 & $11.8(6.2-14.7)$ & \\
\hline & $12 \mathrm{w}$ & 25 & $12.1(7.6-13.7)$ & \\
\hline Initial vs $12 \mathrm{w} \mathrm{Q}_{\max }$ & & & & $<0.001$ \\
\hline
\end{tabular}

IPSS = international prostate symptom score., In the silodosin group, there was a significant reduction in IPSS $(P=<0.001)$ and a significant increase in $\mathrm{Q}_{\max }(P=<0.001)$ between the initial and 12-week assessment (table 3)

Table 3: Effect of silodosin on IPSS and uroflowmetry

\begin{tabular}{llll}
\hline & & $\mathbf{N}$ & mean \pm SD \\
\hline IPSS & Initial & 25 & $16.8 \pm 5$ \\
& $4 \mathrm{w}$ & 25 & $13.3 \pm 3.7$ \\
& $8 \mathrm{w}$ & 25 & $11 \pm 2.9$ \\
Initial vs 12 w IPSS & $12 \mathrm{w}$ & 25 & $10.2 \pm 2.3$ \\
$\mathrm{Q}_{\max }$ & & & \\
(ml/s) & Initial & 25 & $10.9(1.9-19.8)$ \\
Initial vs 12 w Q & $4 \mathrm{w}$ & 25 & $11.6(5.5-14.6)$ \\
& $8 \mathrm{w}$ & 25 & $12.4(7.8-14.9)$ \\
\hline
\end{tabular}

IPSS = international prostate symptom score., We also compared the effects of tamsulosin and silodosin. The analysis showed that there were no significant differences in IPSS and uroflowmetry between the tamsulosin and silodosin groups at the initial assessment or after 4, 8, or $12 \mathrm{w}$ of treatment (table 4).

Table 4: Comparison of IPSS and uroflowmetry between tamsulosin and silodosin groups

\begin{tabular}{lllll}
\hline & & Tamsulosin median (min-max) & Silodosin median (min-max) & $P$ \\
\hline IPSS & Initial & $15(9-29)$ & $17(8-26)$ & 0.808 \\
& $4 \mathrm{w}$ & $11(8-22)$ & $14(8-19)$ & 0.483 \\
& $8 \mathrm{w}$ & $9(7-17)$ & $11(7-17)$ & 0.190 \\
$\mathrm{Q}_{\max }(\mathrm{ml} / \mathrm{s})$ & $12 \mathrm{w}$ & $9(7-15)$ & $10(7-15)$ & 0.186 \\
& Initial & $10.1(4.1-13.4)$ & $10.9(1.9-19.8)$ & 0.290 \\
& $4 \mathrm{w}$ & $11.5(5.7-14.5)$ & $11.6(5.5-14.6)$ & 0.432 \\
& $8 \mathrm{w}$ & $11.8(6.2-14.7)$ & $12.4(7.8-14.9)$ & 0.382 \\
& $12 \mathrm{w}$ & $12.1(7.6-13.7)$ & $11.9(8-13.8)$ & 0.969 \\
\hline
\end{tabular}

IPSS $=$ international prostate symptom score. 


\section{DISCUSSION}

Many $\alpha 1-A R s$, including tamsulosin, prazosin, terazosin, and alfuzosin, have been used to treat BPH associated with moderate to severe LUTS [11] and have been clinically demonstrated to improve LUTS symptoms in BPH. The first-generation $\alpha 1$-ARs frequently cause the side effect of orthostatic hypotension [10], and so new $\alpha 1$-ARs with fewer side effects are needed. Silodosin, a new indolin derivative, is a new-generation $\alpha 1$ AR developed in Japan during the 1990s [12].

The present study showed a significant decrease in IPSS and increase in Qmax between the initial assessment and the 12-week follow-up for both tamsulosin and silodosin groups. The median IPSS of the tamsulosin group was reduced to 7 and that of the silodosin group to 6 after $12 \mathrm{w}$ of treatment, although the difference between groups was not significant. The median Qmax was significantly increased by $1 \mathrm{ml} / \mathrm{s}$ in the silodosin group and $2 \mathrm{ml} / \mathrm{s}$ in the tamsulosin group after $12 \mathrm{w}$ of drug administration, although again, there was no significant difference between silodosin and tamsulosin groups.

Several studies have compared the efficacy of tamsulosin and silodosin in BPH. Chapple et al. showed a reduction of IPSS in tamsulosin and silodosin groups compared with a placebo group [13], although there was no significant difference in IPSS between the treatment groups at the end of the study. Natarajan et al. showed that there was no significant difference between groups treated with silodosin and tamsulosin after $12 \mathrm{w}$ of administration [5]. Takeshita et al. also showed a decrease in IPSS after $4 \mathrm{w}$ of tamsulosin and silodosin administration, and there was no significant difference between the groups [14]. Pande et al. also showed that there was no significant difference in IPSS between tamsulosin and silodosin groups at any follow-up [15]. A clinical trial by Manohar et al. that compared tamsulosin, alfuzosin, and silodosin also showed that there was no significant difference in IPSS between groups after 12 $\mathrm{w}$ of administration. Therefore, the findings of the present study are consistent with many reports that there is no significant difference between the decrease in IPSS induced by treatment with tamsulosin or silodosin for up to $12 \mathrm{w}[5,13-16]$.

However, the findings for $\mathrm{Q}_{\max }$ are less consistent. Chapple et al. reported that both tamsulosin and silodosin induced an increase in $Q_{\max }$ between the initial assessment and after $12 \mathrm{w}$ of treatment, and that there was no significant difference between the drugs [13]. However, Takeshita et al. found that the only improvement in $Q_{\max }$ occurred within the first month of silodosin administration, and that tamsulosin had no effect on $\mathrm{Q}_{\max }$ [14], while Pande et al. reported no improvement of $Q_{\max }$ between initial and 12-week assessments [15]. A clinical trial by Manohar et al. found that $1 \mathrm{w}$ of administration of silodosin had a greater effect on maximal flow compared with $1 \mathrm{w}$ of tamsulosin or alfuzosin [16]. The $\mathrm{Q}_{\max }$ was also increased after 4 and $12 \mathrm{w}$ of treatment, although the difference between silodosin, tamsulosin, and alfuzosin groups was not significant. The findings of the present study are consistent with those of Chapple $e t$ al. and Manohar et al.

This study has several limitations. Ultrasound-guided measurement of prostate volume before and after drug administration was not performed in this study because of limited resources. The lack of resources also limited the number of subjects, which affected the study because the complication rate associated with drug administration could not be assessed. The ethnic profile of the subjects remains to be investigated. However, this study was the first clinical trial to compare silodosin and tamsulosin efficacy for treating BPH in Indonesian patients and could be a reference study for further research in Indonesia.

\section{CONCLUSION}

Silodosin had similar efficacy to tamsulosin for decreasing IPSS and increasing $\mathrm{Q}_{\max }$. Silodosin could be used to control LUTS symptoms in patients with BPH in Indonesia. Further study is required to investigate the efficacy and complication rate of silodosin.

\section{ACKNOWLEDGEMENT}

The authors would like to thank the staff of Urology that took part in the care of these patients. This article was presented at The 3rd International Conference and Exhibition on Indonesian Medical
Education and Research Institute (ICE on IMERI 2018), Faculty of Medicine, Universitas Indonesia, Jakarta, Indonesia. We thank the 3rd ICE on IMERI Committee who had supported the peer review and manuscript preparation before submitting to the journal.

\section{AUTHORS CONTRIBUTIONS}

All the author have contributed equally

\section{CONFLICT OF INTERESTS}

\section{Declared none}

\section{REFERENCES}

1. Mochtar CA, Umbas R, Soebadi DM, Rasyid N, Noegroho BS, Poernomo BB, et al. Pedoman penatalaksanaan klinis pembesaran prostat jinak (Benign prostatic hyperplasia/BPH). Indones Urol Assoc; 2015. p. 1-27.

2. Egan KB. The epidemiology of benign prostatic hyperplasia associated with lower urinary tract symptoms: prevalence and incident rates. Urol Clin North Am 2016;43:289-97.

3. Tobergte DR, Curtis S. EAU guidelines 2016. J Chem Inf Model 2013;53:1689-99.

4. Platz EA, Joshu CE, Mondul AM, Peskoe SB, Willett WC, Giovannucci E. Incidence and progression of lower urinary tract symptoms in a large prospective cohort of United States men. J Urol 2012;188:496-501.

5. Natarajan B, Kalra Y. Silodosin versus tamsulosin in symptomatic benign prostatic hyperplasia-our experience. IOSR J Pharm 2015;5:8-10.

6. Silva J, Silva CM, Cruz F. Current medical treatment of lower urinary tract symptoms/BPH: do we have a standard? Curr Opin Urol 2014;24:21-8.

7. Takao T, Tsujimura A, Kiuchi H, Matsuoka Y, Miyagawa Y, Nonomura $\mathrm{N}$, et al. Early efficacy of silodosin in patients with lower urinary tract symptoms suggestive of benign prostatic hyperplasia. Int J Urol 2008;15:992-6.

8. Tatemichi S, Kobayashi K, Yokoi R, Maruyama K, Hoyano Y, Kobayashi M, et al. Comparison of the effects of four alpha1adrenoceptor antagonists on ejaculatory function in rats. Urology 2012;80:486, e9-16.

9. Miyakita H, Yokoyama E, Onodera Y, Utsunomiya T, Tokunaga $\mathrm{M}$, Tojo T, et al. Short-term effects of crossover treatment with silodosin and tamsulosin hydrochloride for lower urinary tract symptoms associated with benign prostatic hyperplasia. Int J Urol 2010;17:869-75.

10. Kawabe K, Yoshida M, Homma Y. Silodosin, a new alpha1Aadrenoceptor-selective antagonist for treating benign prostatic hyperplasia: results of a phase III randomized, placebo-controlled, double-blind study in Japanese men. BJU Int 2006;98:1019-24.

11. Gravas S, Bach T, Drake M, Gacci M, Gratzke C, Herrmann TRW, et al. EAU guidelines on non-neurogenic male LUTS including benign prostatic obstruction (BPO). Eur Assoc Urol 2017;2:129-46.

12. Yoshida M, Kudoh J, Homma Y, Kawabe K. Safety and efficacy of silodosin for treatment of benign prostatic hyperplasia. Clin Intervent Aging 2011;6:161-72.

13. Chapple CR, Montorsi F, Tammela TLJ, Wirth M, Koldewijn E, Fernandez EF. Silodosin therapy for lower urinary tract symptoms in men with suspected benign prostatic hyperplasia: results of an international, randomized, double-blind, placebo-and activecontrolled clinical trial performed in Europe. Eur Urol 2011;59:342-52.

14. Takeshita H, Moriyama S, Arai Y, Washino S, Saito K, Chiba K, et al. Randomized crossover comparison of the short-term efficacy and safety of single half-dose silodosin and tamsulosin hydrochloride in men with lower urinary tract symptoms secondary to benign prostatic hyperplasia. LUTS 2016;8:38-43.

15. Pande S, Hazra A, Kundu AK. Evaluation of silodosin in comparison to tamsulosin in benign prostatic hyperplasia: a randomized controlled trial. Indian J Pharmacol 2014;46:601-7.

16. Manohar CMS, Naggabhushana M, Karthikeyan VS, Sanjay RP, Kamath AJ, Keshavamurty R. Safety and efficacy of tamsulosin, alfuzosin or silodosin as monotherapy for LUTS in BPH-a doubleblind randomized trial. Cent European J Urol 2017;70:148-53. 